\title{
Pressure-induced tricritical point in the ferroelectric phase transition of $\mathrm{KH}_{2} \mathrm{PO}_{4}$
}

\author{
Arthur B. Western, ${ }^{*}$ Alan G. Baker, ${ }^{\dagger}$ Charles R. Bacon, ${ }^{\ddagger}$ and V. Hugo Schmidt \\ Department of Physics, Montana State University, Bozeman, Montana 59717 \\ (Received 20 December 1976; revised manuscript received 31 January 1978)
}

\begin{abstract}
Measurement of the net polarization charge of two $\mathrm{KH}_{2} \mathrm{PO}_{4}$ crystals as a function of temperature, applied electric field, and hydrostatic pressure indicates the existence of a tricritical point in the $(2.3 \pm 0.3)$-kbar pressure range. This result is based upon static measurements of the polarization response to applied dc field in a $0.5-\mathrm{K}$ neighborhood of the ferroelectric transition at pressures of $0,1,2,2.4$, and $3 \mathrm{kbar}$. Unlike ${ }^{3} \mathrm{He}-$ ${ }^{4} \mathrm{He}$ mixtures and some metamagnets, for $\mathrm{KH}_{2} \mathrm{PO}_{4}$ the field which gives rise to the "wing" structure of the tricritical point is experimentally available. For each of the five pressures the paraelectric region is well described by the Landau equation of state, $E=A_{0}\left(T-T_{0}\right) P+B P^{3}+C P^{5}$, to within $0.05 \mathrm{~K}$ of the transition temperature. The exponent $\delta$ derived from our data at $3 \mathrm{kbar}$ is in the crossover region between the critical value of 3 and the tricritical value of 5 predicted by Landau theory. At $2.4 \mathrm{kbar}$ our derived value of $\delta$ is consistent with the tricritical value of 5 . Analysis of the data along lines of constant polarization, which are here called "isopols," indicates that the transition is first order at 0 and 1 kbar with the critical field decreasing from $183 \pm 60 \mathrm{~V} / \mathrm{cm}$ at $0 \mathrm{kbar}$ to $43 \pm 13 \mathrm{~V} / \mathrm{cm}$ at $1 \mathrm{kbar}$. At $3 \mathrm{kbar}$ the $B$ coefficient is positive which indicates a second-order transition. This observation of a change in the order of the transition is supported by a change in the behavior of the isothermal dielectric susceptibility which has a maximum for $E>0$ at $0.5 \mathrm{kbar}$ and at $E=0$ at $3 \mathrm{kbar}$.
\end{abstract}

\section{INTRODUCTION}

A tricritical point was defined by Griffiths ${ }^{1}$ as a point where three lines of critical points meet in a space of thermodynamic fields. For ${ }^{3} \mathrm{He}-{ }^{4} \mathrm{He}$ mixtures, the magnetic transition in $\mathrm{FeCl}_{2}$, and the structural transition in $\mathrm{NH}_{4} \mathrm{Cl}$, one of the critical lines borders a first-order coexistence surface in a plane formed by two experimentally realizable. fields. The other two critical lines border firstorder surfaces which appear as symmetric "wings" to the first plane, but the ordering field necessary to examine the wing structure is not experimentally available. For example, in the antiferromagnetic case a staggered magnetic field with opposite direction on each sublattice would have to be applied. ${ }^{2}$

In the experimentally accessible zero-orderingfield plane the tricritical point is identified as the point where a first-order coexistence line (actually a line of triple points) changes to a secondorder line. This latter possibility was discussed much earlier by Landau ${ }^{3}$ who argued that a secondorder coexistence line separating two phases of different symmetry can not end in a point. Rather, he proposed that a second-order phase boundary could terminate by changing continuously into a first-order line. Landau's phenomenological description is based upon the expansion of the free energy as a Taylor polynomial in the order parameter. In the case most often discussed the free energy has the form

$$
F=\frac{1}{2} A_{0}\left(T-T_{0}\right) P^{2}+\frac{1}{4} B P^{4}+\frac{1}{6} C P^{6}+\frac{1}{8} D P^{8}+\cdots,
$$

where $T$ is the temperature and $P$ is the order parameter. If terms of order $P^{6}$ and greater are positive definite then the order of the transition described by Eq. (1) depends upon the $\operatorname{sign}$ of $B$, being first order for $B<0$ and second order for $B>0$. If $B$ changes smoothly from positive to negative as a function of some external parameter, then a "Landau tricritical point" is realized at $B=0$.

The present work was motivated by the conjecture of $\mathrm{Schmidt}^{4}$ that the ferroelectric crystal $\mathrm{KH}_{2} \mathrm{PO}_{4}(\mathrm{KDP})$ would exhibit a tricritical point with experimentally accessible wings in the space of temperature, electric field, and hydrostatic pressure. Since that time, Peercy ${ }^{5}$ has shown that another ferroelectric, SbSI, has a tricritical point near $235 \mathrm{~K}$ and $1.40 \mathrm{kbar}$. In this paper we report on static measurements of the polarization of two $\mathrm{KH}_{2} \mathrm{PO}_{4}$ single crystals which indicate that such a tricritical point does exist. Thus far it has been possible to describe the crystal behavior in terms of the Landau phenomenological description of a tricritical point.

In Sec. II we review recent work by others concerning the order of KDP transition at ambient pressure and the effect of hydrostatic pressure on the transition temperature. Section III contains a description of our experimental configuration. Section IV is an introduction to the analysis of data along lines of constant polarization which we call "isopols." In Sec. V we present isopol data and results for the best fit coefficients in the Landau expression for free energy. Section VI compares measured critical exponents with predictions of 
Landau theory. Section VII summarizes our findings and compares them with other recent results.

\section{RECENT RELEVANT EXPERIMENTS}

Prior to 1969 the ferroelectric transition in KDP at ambient pressure was generally thought to be second order. ${ }^{6}$ Recent results indicate that it is in fact first order but quite close to being second order. This opinion is now supported by a number of experiments: Strukov et al. ${ }^{7}$ exploited KDP's large electrocaloric effect and measured the temperature change produced by the sudden application of an electric field. Sidnenko and Gladkii ${ }^{8}$ measured the polarization versus temperature of the crystal in various electric fields. Vallade ${ }^{9}$ measured the temperature dependence of the polarization of KDP by optical birefringence. Okada and Sugié and others have studied the KDP transition extensively. They have reported on the temperature sweep rate dependence of the thermal hysteresis, ${ }^{10}$ the difference between the adiabatic and isothermal dielectric constant, ${ }^{11}$ and quasistatic measurements of the polarization versus applied field at constant temperature. ${ }^{12}$ Reese and others have studied the transition in calorimetric ${ }^{13}$ and electrocaloric ${ }^{14}$ experiments. The most recent results of all of these groups are in fair agreement as to the coordinates of the critical point at the end of the first-order line $(200-300 \mathrm{~V} / \mathrm{cm})$ and the fact that KDP obeys the phenomenological theory of Landau to within at least $0.1 \mathrm{~K}$ of the transition temperature.

There are, however, three experiments de- scribed in the literature which yielded markedly different results. The first of these is an $x$-ray dilatometric study by Kobayashi et al. ${ }^{15}$ who found a critical field of $8500 \mathrm{~V} / \mathrm{cm}$, much higher than the critical fields of $200-300 \mathrm{~V} / \mathrm{cm}$ found in the experiments described above. Matsuda and $\mathrm{Abe}^{16}$ calculated the $B$ coefficient in the Landau free energy from measurements of the third harmonic of a $1-\mathrm{kHz}$ ac electric field applied to the crystal. Their value is two orders of magnitude larger (in absolute value) than reported in the papers cited earlier. Finally, Eberhard and Horn ${ }^{17}$ studied the thermal hysteresis of the transition at various applied fields and concluded $E_{\text {cr }}=6500 \mathrm{~V} / \mathrm{cm}$. There is reason to believe ${ }^{18}$ that this value should be revised downward closer to $300 \mathrm{~V} / \mathrm{cm}$.

The latest published results of all of these workers are displayed in Table I along with our previous results for two samples ${ }^{19,20}$ and our presently reported results for a third sample. The parameters in Table I refer to the equation of state

$$
E=A_{0}\left(T-T_{0}\right) P+B P^{3}+C P^{5}+D P^{7}
$$

which follows from differentiating the free energy given in Eq. (1) with respect to $P$ at constant temperature and pressure. Here $E$ is the electric field, $T$ is the temperature, and $P$ is the polarization which acts as the order parameter for the transition. Values for the coordinates of the critical point at the termination of the first-order line have been calculated using

$$
\begin{aligned}
& T_{\mathrm{cr}}-T_{0}=A_{\mathrm{cr}} / A_{0}=-\left(3 B P_{\mathrm{cr}}^{2}+5 C P_{\mathrm{cr}}^{4}+7 D P_{\mathrm{cr}}^{6}\right) / A_{0}, \\
& E_{\mathrm{cr}}=A_{\mathrm{cr}} P_{\mathrm{cr}}+B P_{\mathrm{cr}}^{3}+C P_{\mathrm{cr}}^{5}+D P_{\mathrm{cr}}^{7},
\end{aligned}
$$

TABLE I. Recent published values of the parameters in the free energy $F=\frac{1}{2} A_{0}\left(T-T_{0}\right) P^{2}+\frac{1}{4} B P^{4}$ $+\frac{1}{6} C P^{6}+\frac{1}{8} D P^{8}$ and derived coordinates of the critical point at ambient pressure. ${ }^{\mathrm{a}}$

\begin{tabular}{cllllll}
\hline \hline & $\begin{array}{c}A \\
\text { Reference }\end{array}$ & \multicolumn{1}{c}{$\begin{array}{c}B \\
\left(10^{-3} \mathrm{esu}\right)\end{array}$} & \multicolumn{1}{c}{$\begin{array}{c}C \\
\left(10^{-11} \mathrm{esu}\right)\end{array}$} & $\begin{array}{c}D \\
\left(10^{-19} \mathrm{esu}\right)\end{array}$ & $\begin{array}{c}E_{\text {cr }} \\
\left(10^{-27} \mathrm{esu}\right)\end{array}$ & $\begin{array}{c}T_{\text {cr }}-T_{0} \\
(\mathrm{~V} / \mathrm{cm})\end{array}$ \\
\hline 7 & 3.9 & -1.9 & 6.3 & 0 & 120 & 0.07 \\
8 & $3.8 \pm 0.1$ & $-3.0 \pm 0.8$ & $6.5 \pm 1.1$ & 0 & 370 & 0.16 \\
& $3.8 \pm 0.1$ & $-0.5 \pm 0.3$ & 0 & $3.8 \pm 0.4$ & 87 & 0.036 \\
9 & 3.9 & $-0.54 \pm 0.05$ & 0 & $2.85 \pm 0.10$ & 124 & 0.046 \\
& 3.9 & $-1.85 \pm 0.25$ & $3.3 \pm 0.5$ & $0.87 \pm 0.5$ & 280 & 0.11 \\
12 & $4.2 \pm 0.1$ & $-1.9 \pm 0.1$ & $5.4 \pm 0.4$ & 0 & 160 & 0.07 \\
14 & $(3.81)$ & -0.44 & 0 & 2.96 & 84 & 0.055 \\
15 & $(3.86)$ & -11.9 & 11.0 & 0 & 8500 & 1.50 \\
16 & $\ldots$ & -110 & $\ldots$ & $\ldots$ & $\ldots$ & $\ldots$ \\
17 & $(7.3)^{\mathrm{b}}$ & -2.2 & 0.6 & 0 & 6500 & 0.51 \\
Our work & & & & & & \\
19 (Sample 1) & $4.3 \pm 0.2$ & $-2.35 \pm 0.4$ & $5.91 \pm 1.5$ & 0 & $232 \pm 70$ & $0.10 \pm 0.03$ \\
20 (Sample 2) & $4.0 \pm 0.2$ & $-1.48 \pm 0.2$ & $3.1 \pm 0.4$ & 0 & $186 \pm 60$ & $0.08 \pm 0.03$ \\
(Sample 3) & $3.91 \pm 0.04$ & $-1.26 \pm 0.05$ & $3.2 \pm \pm 0.1$ & 0 & $123 \pm 18$ & $0.057 \pm 0.007$ \\
\hline \hline
\end{tabular}

${ }^{a}$ Numbers in parentheses were obtained from a source other than the primary reference.

${ }^{\mathrm{b}}$ Reference 18 . 
where $P_{\text {cr }}$ is given by

$$
\begin{aligned}
& P_{\mathrm{cr}}^{2}=(5 C / 21 D)\left[\left(1-63 B D / 25 C^{2}\right)^{1 / 2}-1\right], \\
& \quad C \neq 0, \quad D \neq 0, \\
& P_{\mathrm{cr}}^{2}=(-B / 7 D)^{1 / 2}, C=0, D \neq 0, \\
& P_{\mathrm{cr}}^{2}=-3 B / 10 C, C \neq 0, D=0 .
\end{aligned}
$$

There has been considerable discussion ${ }^{7,12,14}$ concerning the proper form for the saturation function of the free energy, i.e., which of the terms of order greater than $P^{3}$ should be included in Eq. (2) in order to give the "best" description of the large polarization response of KDP. At present a quantitative comparison of the results of various authors is not possible. It would be particularly useful if the method of orthogonal polynomials ${ }^{21}$ were applied to such polarization data so that statistically quantitative statements could be made regarding the degree of fit of various forms for the saturation function. In our experiment the reduction of the residual sum of squares resulting from the addition of a $D P^{7}$ term wás not statistically significant. This was expected as polarization values obtained in the paraelectric region were not large enough to resolve higher-order terms.

The effect of hydrostatic pressure on the transition temperature of KDP has been studied using neutron scattering by Umebayashi et al. ${ }^{22}$ and in dielectric experiments by Hegenbarth and Ullwer ${ }^{23}$ and by Samara. ${ }^{24}$ In these experiments the temperature resolution was not sufficient to differentiate between the Curie-Weiss temperature $T_{0}$ and the actual transition temperature $T_{c}$, so we compare the above measurements of $d T_{c} / d p$ with our findings for $d T_{0} / d p$ in Table II. The value of $d T_{0} / d p=4.54 \pm 0.05 \mathrm{~K} / \mathrm{kbar}$ obtained in this work is in agreement with those of Umebayashi et al. ${ }^{22}$ and Samara. ${ }^{24}$

\section{EXPERIMENTAL}

The preparation of the first crystal and results obtained from it at ambient pressure have been described previously. ${ }^{19}$ The second KDP single crystal was obtained from Cleveland Crystals, Inc. ${ }^{25}$ and stored in a vial with $\mathrm{CaSO}_{4}$ desiccant for 20

TABLE II. Summary of reports of the effect of pressure on the ferroelectric transition temperature of $\mathrm{KH}_{2} \mathrm{PO}_{4}$.

\begin{tabular}{cll}
\hline \hline Reference & $\begin{array}{l}d T_{0} / d p, \\
(\mathrm{~K} / \mathrm{kbar})\end{array}$ & \multicolumn{1}{c}{ Method } \\
\hline 22 & $-4.52 \pm 0.06$ & Neutron scattering \\
23 & -5.6 & Dielectric constant \\
24 & $-4.6 \pm 0.1$ & Dielectric constant \\
Present work & $-4.54 \pm 0.05$ & Isopols \\
\hline
\end{tabular}

months. Crystal dimensions were $1 \times 1 \times 0.2 \mathrm{~cm}^{3}$, the large faces being perpendicular to the ferroelectric $c$ axis. Chrome-gold electrodes had been evaporated onto the surface by the supplier. The 0.0025-in. diameter Solid Copperweld center wires (copperplated steel wires) of Type $A$ Ultraminiature Coaxial $\mathrm{Cable}^{26}$ were attached to the evaporated electrode faces by means of silver paint ${ }^{27}$ applied as small $\left(\sim 4 \mathrm{~mm}^{2}\right)$ dots on either side. Five coats were used in an effort to increase the strength of the bond as these lead wires were the sole support for the crystal. Thus the only stress on the unpressurized freely hanging crystal was that caused by its own weight.

A corner of the second crystal broke off during apparatus disassembly after the end of the $3-\mathrm{kbar}$ run. The remaining work was carried out with a third crystal of the same dimensions and suspended in the same way as the second crystal. This crystal was obtained from Cleveland Crystals, Inc. ${ }^{25}$ about two months before it began service as a sample.

In order to check crystal quality the small signal $(0.05 \mathrm{~V} / \mathrm{cm})$ ac dielectric constant $\epsilon$ was measured at $1 \mathrm{kHz}$. From $T_{\mathbf{s}}+0.3 \mathrm{~K}$ to $T_{c}$ a straight line was obtained for $\epsilon^{-1}$ vs $T$ in accordance with the CurieWeiss law. The second crystal showed a slight decrease in $\epsilon$ at $40 \mathrm{mK}$ above $T_{0}$, then the dielectric constant rose to 360000 after which it stayed constant for at least $0.2 \mathrm{~K}$ below $T_{0}$. The third crystal had a peak dielectric constant of 430000 .

Conventional wisdom attributes the above behavior to domain wall motion. Bornarel, Fousková, Guyon, and Lajzerowicz ${ }^{28}$ showed that the dielectric constant $12 \mathrm{~K}$ below $T_{c}$ could be reduced from $4.5 \times 10^{4}$ to $1.4 \times 10^{4}$ by reducing the size of the applied ac fields from $2 \mathrm{~V} / \mathrm{cm}$ to $0.5 \mathrm{~V} / \mathrm{cm}$. This would seem to support the domain wall explanation. In the course of this investigation ac fields as small as $0.005 \mathrm{~V} / \mathrm{cm}$ were used in a $0.3 \mathrm{~K}$ neighborhood of $T_{c}$ with slight reduction in dielectric constant, but no change in the temperature dependence. If domain wall motion is responsible for the high dielectric constant in the ferroelectric region, the walls are very mobile indeed immediately below $T_{c}$.

The sample was suspended in a beryllium-copper $^{29}$ pressure vessel (PV) surrounded by two concentric cylindrical cans with 0.25 -in. diameter high-pressure tubing acting as the central connecting axis. A schematic drawing of the cryostat is shown in Fig. 1. The region of the cryostat external to the PV was evacuated to $10^{-4}$ Torr or better and the entire assembly immersed in a dewar of liquid nitrogen. The point where the pressure tubing passes through the inner copper shield can, marked $C$ in Fig. 1, was held at a fixed $( \pm 0.1 \mathrm{~K})$ 


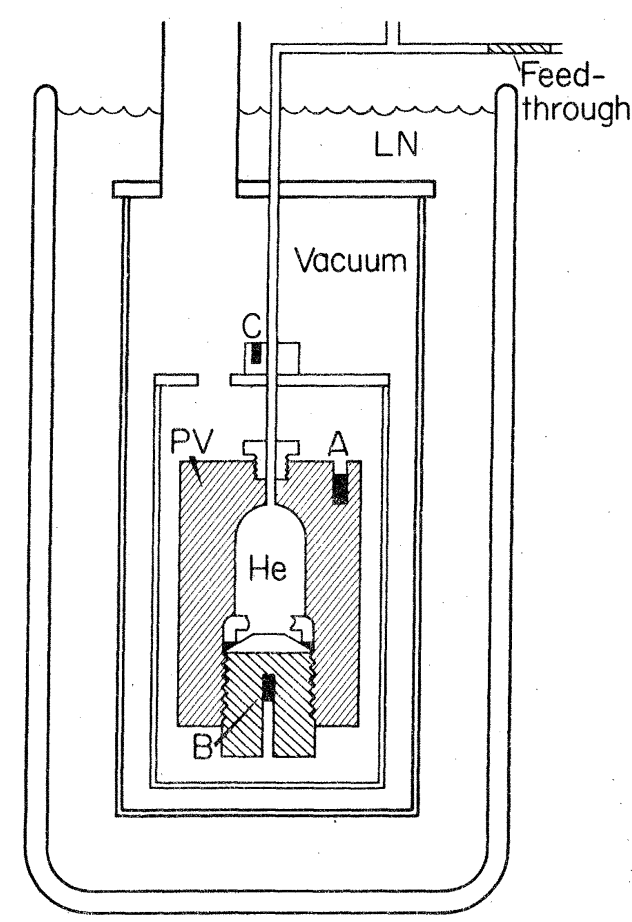

FIG. 1. Schematic drawing of cryostat used to control pressure vessel (PV) temperature. A copper-constantan thermocouple was located at $C$, and capacitance thermometers at $A$ and $B$.

temperature about $3 \mathrm{~K}$ below the $\mathrm{PV}$ temperature by means of heater current controlled by a feedback amplifier using a copper--constantan thermocouple located at point $C$ as the temperature sensor.

The temperature of the PV itself was regulated using a commercial temperature controller ${ }^{30} \mathrm{em}-$ ploying as the temperature sensor a glass-encapsulated capacitor with strontium titanate dielectric, ${ }^{31}$ located at point $A$ of Fig. 1 . The temperature of the sample was assumed to be the same as that of a second capacitor embedded in the closure plug of the PV, point $B$ in Fig. 1. Care was taken to maintain a constant temperature difference between the two sensors over the temperature intervals studied. The temperature sensors were calibrated in situ against copper-constantan thermocouples using a Leeds and Northrup K-5 Potentiometer and distilled water ice bath. In the temperature range studied the controller has a temperature resolution of $\pm 2 \mathrm{mK}$ and the pressure-vessel temperature can be held stable with this precision for arbitrary lengths of time.

The sample was pressurized with helium gas using a diaphragm pump and intensifier. The pressure was determined using a Cary-Foster ${ }^{32}$-type Wheatstone bridge to measure the resistance of a thermostated manganin cell. The resistance was measured with an accuracy equivalent to $\pm 50 \mathrm{psi}$ and the stability monitored with a precision of \pm 1 psi. As Table II shows, the crystal transition temperature changes as a function of pressure. In order to maintain the crystal transition temperature constant to $\pm 2 \mathrm{mK}$ the pressure was maintained constant to within \pm 0.5 bar by occasional pumping to offset a leak from the manganin cell. ${ }^{33}$ Such pumping disturbed the equilibrium of the crystal and care was taken to allow the crystal to return to the steady state before resuming measurements. Modeling the pressure-system leaks as an isentropic throttling process indicates that at the maximum pressure leak rate $(\sim 1 \mathrm{bar} / \mathrm{h}$ at $3 \mathrm{kbar})$ the flow work proceeded at a rate of $\sim 1 \mathrm{~mW}$. Since this amount of power is easily exchanged between the pressurized helium gas and the pressure tubing, the finite pressure leak had negligible cooling effect on the crystal. The electrical stability of the pressure-measuring system was monitored at a known pressure of 1 bar and found to be equivalent to \pm 0.7 bar over a period of three days.

Static electric fields were applied to the crystal using a battery and resistive voltage divider. Polarization charge was stored on an $8-\mu \mathrm{F}$ polystyrene capacitor in series with the sample. The resultant voltage was measured with a high-impedance $\left(10^{12} \Omega\right)$ electrometer. ${ }^{34}$ The measurement circuit had an $R C$ time constant of $8 \times 10^{6}$ sec, resulting in a maximum leak rate of $1.1 \%$ / day. Stray charge leakage was maintained below this value by the use of Teflon insulation and careful guarding. Further experimental details may be found elsewhere. ${ }^{35}$

\section{ISOPOLS}

There is a tradition in the literature of measuring polarization $(P)$ as a function of electric field $(E)$ along isotherms, or as a function of temperature $(T)$ along isochamps. Most of the data to be presented here is displayed as a function of $T$ and $E$ along lines of constant polarization, i.e., along isopols. This approach appears to be new and thus the following discussion is devoted to the interpretation of such plots. This discussion is based upon the Landau equation of state given in Eq. (2); however, many of the conclusions based on isopol plots are independent of this equation of state. The reader is cautioned not to equate the validity of all conclusions with the validity of the Landau expansion which is used here merely as a vehicle for introducing the isopol picture.

The equation of state [Eq. (2)] which follows from the Landau free energy given in Eq. (1), when 
rewritten, indicates that the isopols are straight lines in the $T-E$ plane with slopes $\left(A_{0} P\right)^{-1}$ and $E=0$ intercepts $T_{0}-\left(B P^{2}+C P^{4}\right) / A_{0}$; i.e.,

$$
T=\left(A_{0} P\right)^{-1} E+T_{0}-\left(B P^{2}+C P^{4}\right) / A_{0} .
$$

In the limit of small $P$ the $E=0$ intercepts tend to $T_{0}$. If $B$ is negative, when $P$ increases the intercepts rise above $T_{0}$ and then fall as $P$ increases further and the $C P^{4}$ term begins to dominate. For $B$ positive the intercepts simply fall farther and farther below $T_{0}$ as $P$ increases. The case for negative $B$ is shown in Fig. 2 .

The initial increase and subsequent decrease in the $T$ intercepts creates a region where isopols intersect. The first-order line, $F D$ in Fig. 2, liès within the overlap region which is bounded by caustics $A C D B$. Note that $B C E$ is the extension of a line similar to $B D$ in the negative $E$ half plane not shown in Fig. 2. The critical point $D$ is at a vertex of the curvilinear triangle formed by the caustics of intersecting isopols. Isopols are shown as solid lines when they correspond to an absolute minimum of the free energy. After crossing the first-order line they are shown as dashed lines and correspond to local minima of the Landau free energy.

If metastable states, corresponding to local minima are actually manifested by the crystal, mixed

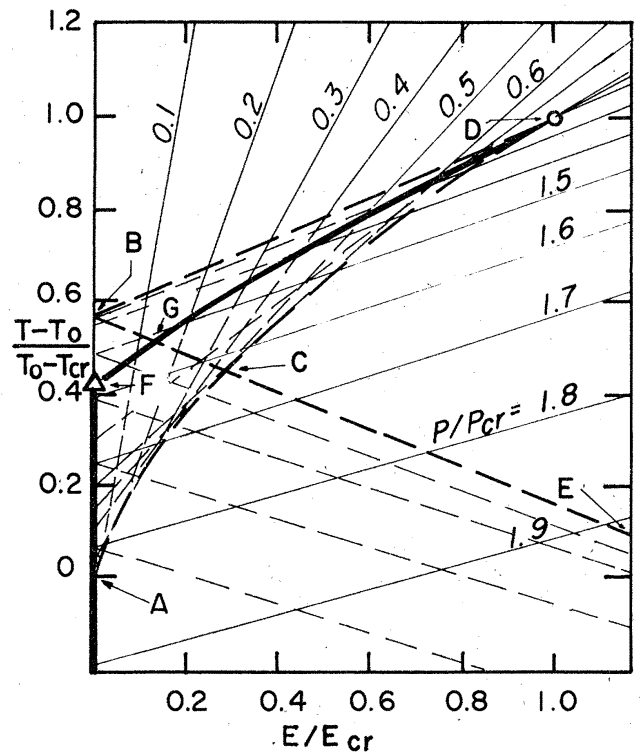

FIG. 2. Phase diagram for a first-order transition as described by $E=A_{0}\left(T-T_{0}\right) P+B P^{3}+C P^{5}$. Triangle is Curie point or triple point. Circle is the critical point. Heavy solid lines indicate first-order transitions. Light solid and dashed lines correspond to isopols for stable and metastable phases, respectively. Heavy dashed lines outline limits of regions of metastability.
TABLE III. Possible mixed phase regions in Fig. 2. Phases corresponding to absolutely stable minima of the free energy are denoted $S$, states corresponding to relative minima are denoted $M$. The polarization is "up" when parallel to the applied $E$ field.

\begin{tabular}{lccc}
\hline \hline Region & Paraelectric & $\begin{array}{c}\text { Ferroelectric } \\
\text { "up" }\end{array}$ & $\begin{array}{c}\text { Ferroelectric } \\
\text { "down" }\end{array}$ \\
\hline$B D G$ & $S$ & $M$ & \\
$C D G$ & $M$ & $S$ & $M$ \\
$B G F$ & $S$ & $M$ & $M$ \\
$A F G C$ & $M$ & $S$ & $M$ \\
$A C E$ & & $S$ & \\
\hline \hline
\end{tabular}

phases exist in the regions of isopol overlap. Denoting regions of polarization parallel to $+E$ as "up," the mixed phase regions may be characterized as in Table III. We have shown elsewhere ${ }^{19}$ that when an experimental isopol crosses a line such as $C E$ in Fig. 2, it changes direction, heading almost vertically downward. This is interpreted as resulting from the creation of mixed phases in the form of ferroelectric domains within the crystal.

In the Landau equation of state the order of the transition is indicated by the sign of the coefficient $B$, being negative for first order and positive for second order. In an isopol plot this difference manifests itself as a convergence or nonconvergence of isopols, respectively. The deduction of the order of the transition from the behavior of isopols is, however, independent of the Landau equation of state. This may be seen by considering an isotherm drawn through an isopol plot just above $T_{\text {cr }}$ (see Fig. 2). If the isopols converge toward an $E \neq 0$ point, then the isotherm will encounter a large change in polarization for a small change in field near the point of convergence, i.e., the dielectric susceptibility will be high. A dielectric susceptibility higher at $E>0$ than at $E=0$ indicates an $E \neq 0$ critical point, and thus a first-order transition at $E=0$ is implied. On the other hand, if the isopols do not converge except for $E=0$ then the dielectric susceptibility is never higher than at $E=0$, and a second-order transition at $E=0$ is indicated.

The advantage of displaying data in isopol plots rather than as electric field dependence of the dielectric susceptibility is the numerical convenience and graphical clarity afforded by fitting straight lines. In addition, deviation from the simple Landau picture becomes obvious when the actual crystal isopols deviate from the high-temperature extrapolations.

Two methods were used for extracting Landau parameters from isopol data. The first method 
begins with calculation of a least-squares straightline fit to paraelectric isopols. From these, $A_{0}$ is obtained from the slopes of the isopols using

$$
A_{\mathrm{o}}=(\partial E / \partial T)_{P} / P \text {. }
$$

An approximate value of $T_{0}$ is found from a plot of the extrapolated $T(E=0)$ intercepts versus $P^{2}$ for the three smallest isopols. This plot is a straight line and should extrapolate to $T_{0}$. The parameters $B$ and $C$ are then deduced from the intercept and slope respectively of a graph of $-A_{0}\left(T-T_{0}\right) / P^{2}$ vs $P^{2}$. In practice $T_{0}$ is then varied a small amount (within the experimental error) to produce the best straight line on this graph. In this latter procedure, points from the higher-polarization isopols are heavily weighted owing to the large scatter produced on this type of graph by the low-polarization isopols for which $T-T_{0}$ is quite small.

The second method begins with calculation of the average temperature $\left\langle T_{i}\right\rangle$ and average field $\left\langle E_{i}\right\rangle$ for the data points for each isopol. The $A_{0 i}$ for the various isopols are then calculated as described above, and their weighted average $A_{0}$ is obtained. The Landau equation of state predicts that

$$
\left\langle T_{i}\right\rangle=T_{0}+\left\langle E_{i}\right\rangle / A_{0} P_{i}-P_{i}^{2} B / A_{0}-P_{i}^{4} C / A_{0}
$$

for the isopol corresponding to polarization $P_{i}$. These equations for the various isopols are solved simultaneously for the values of $T_{0}, B$, and $C$ which give the best fit to the measured $\left\langle T_{i}\right\rangle$, taking the differences in the standard deviations of the $\left\langle T_{i}\right\rangle$ into account with appropriate weighting factors. Mathematical details of this method appear elsewhere. ${ }^{36}$

The first calculational method was used for the first two crystals, and the second method for the third crystal. Both methods were used for the $3-\mathrm{kbar}$ data for the second crystal and the ambient-pressure data for the third crystal, and the results agreed within experimental error.

\section{EXPERIMENTAL RESULTS}

Isopol data for sample 2 at pressures of 0,1 , and $3 \mathrm{kbar}$ are presented in Figs. 3-5. Each data point was taken as close to equilibrium as possible. Finite pressure leaks necessarily led to finite polarization drift. The largest leak rate was $1 \mathrm{bar} / \mathrm{h}$ at $3 \mathrm{kbar}$ which is equivalent (see Table II) to a temperature drift of $5 \mathrm{mK} / \mathrm{h}$. At "equilibrium" the polarization drift rate was less than $2 \% / \mathrm{h}$ in all cases. Tables IV-VI summarize the best fit Landau parameters. The data can be fit satisfactorily with these Landau parameters at

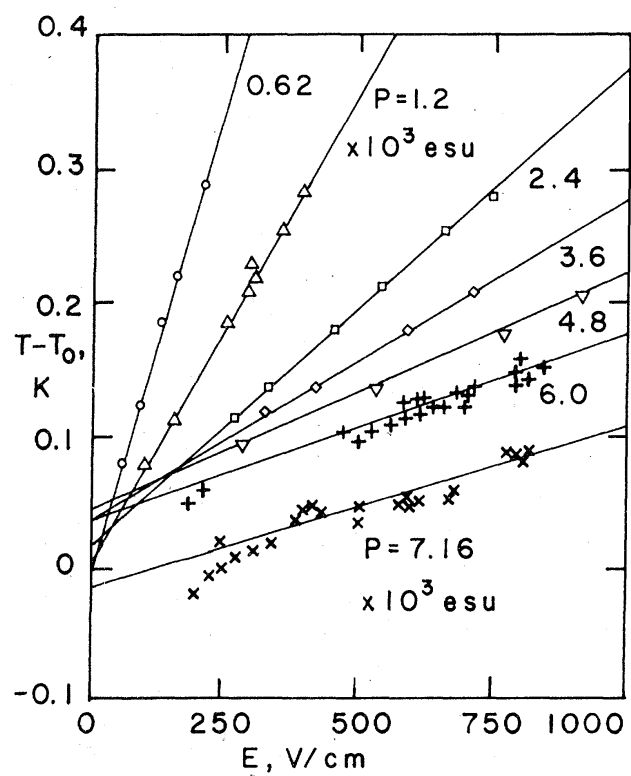

FIG. 3. Isopols at $0 \mathrm{kbar}$. Solid lines are generated from Landau equation of state, $E=A_{0}\left(T-T_{0}\right) P+B P^{3}$ $+C P^{5}$, for best-fit parameters. Intersection of extrapolated isopols indicates a first-order transition.

all three pressures. Crossing of extrapolated isopols in Fig. 3 indicates the first-order nature of the transition graphically. Based upon the bestfit Landau parameters, the ambient-pressure critical field of $186 \pm 60 \mathrm{~V} / \mathrm{cm}$ is reduced to $44 \pm 13$ $\mathrm{V} / \mathrm{cm}$ by $1 \mathrm{kbar}$ of hydrostatic pressure. At 3

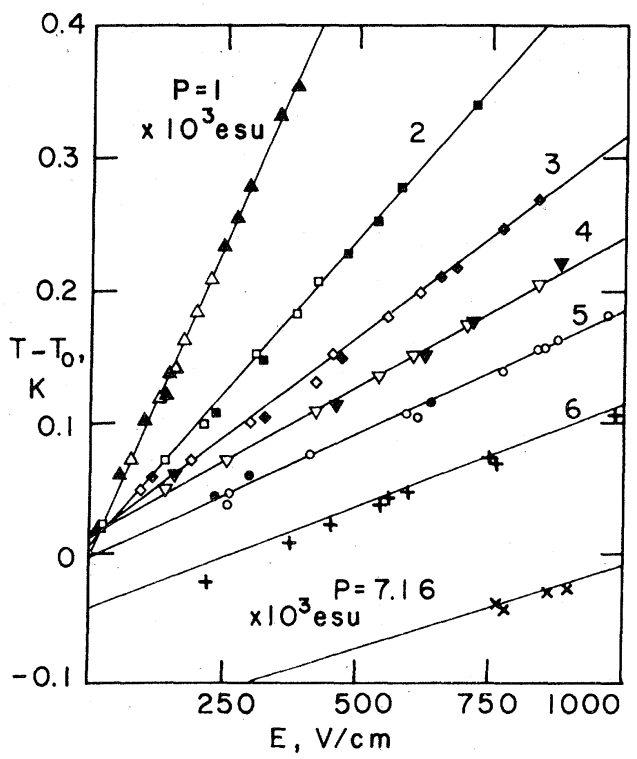

FIG. 4. Isopols at $1 \mathrm{kbar}$. Solid lines are generated from Landau equation of state, $E=A_{0}\left(T-T_{0}\right) P+B P^{3}$ $+C P^{5}$, for best-fit parameters. Intersection of extrapolated isopols indicates a first-order transition. 


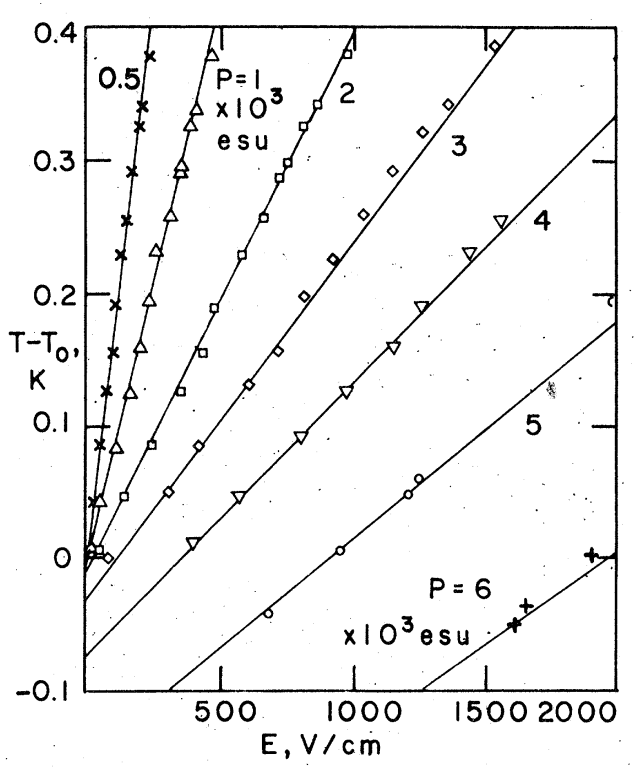

FIG. 5. Isopols at $3 \mathrm{kbar}$. Solid lines are generated from Landau equation of state, $E+A_{0}\left(T-T_{0}\right) P$ $+B P^{3}+C P^{5}$, for best-fit parameters. Extrapolated isopols do not cross, which indicates a second-order transition.

kbar the transition appears to be second order, that is, the extrapolated intercepts all fall below $T_{0}$.

This conclusion regarding the change of the order of the transition is supported by a change in the behavior of the isothermal dielectric constant as deduced from the slope of $P$ vs $E$ traces taken for sample 2 at constant temperature and pressure, and at electric field sweep rates of $240 \mathrm{~V} / \mathrm{cm} \mathrm{h}$. At $0.5 \mathrm{kbar}$ the maximum dielectric constant occurred at $300 \mathrm{~V} / \mathrm{cm}$ while at $3 \mathrm{kbar}$ the dielectric constant was highest at $E=0$. Landau parameters and critical fields deduced from such plots are in general. agreement with those extracted from isopol data, but the values appear to depend upon the electric field sweep rate. Nonetheless, we consider the difference in shape, as shown in Fig. 6, to be highly significant. (See Ref. 12 for details concerning this technique.)

Additional isopol data were obtained with sample 3 at pressures of 0,2 , and $2.4 \mathrm{kbar}$. Graphs of these isopols are not shown here, but the fit to the Landau parameters is as good as for the sample 2 data shown in Figs. 3-5. The best fit Landau parameters for the sample 3 isopols are given in Tables VII-IX. Table IX shows an apparently systematic decrease in $A_{0}$ from run to run for the three runs having total duration of six weeks. Such systematic decrease could be caused by increase of effective contact area, but there was no visual evidence of defective contacts after the runs, so the origin of this systematic variation is unknown. The best fit Landau parameters $A_{0}, B$, and $C$ reported previously ${ }^{19,20}$ for samples 1 and 2 and reported now ${ }^{37}$ for sample 3 are presented in Table $\mathrm{X}$. The $B$ and $C$ values are not dependent upon the values assigned to $A_{0}$, and were determined by the methods described in Sec. IV. The results for $B$ as a function of pressure $p$ are shown in Fig. 7 for all three samples. A least-squares linear $\mathrm{fit}^{38}$ to all seven points yields $B=0$ at $2.18 \mathrm{kbar}$, while linear fits to the sample 2 and sample 3 points give 1.94 and $3.02 \mathrm{kbar}$, respectively. If a quadratic term is included, the corresponding pressures are $2.36,2.11$, and $2.42 \mathrm{kbar}$. Based on these results we consider the tricritical pressure to be $2.3 \pm 0.3 \mathrm{kbar}$.

\section{CRITICAL EXPONENTS}

From the isopol plots in Figs. 3-5 we have seen that $\mathrm{KH}_{2} \mathrm{PO}_{4}$ obeys Landau theory in the regions of

TABLE IV. Data for Sample No. 2 at 0.0016 kbar. Here and in Tables V - IX, $\Delta=\left[\left(A_{0}-\overline{A_{0}}\right)^{2} /(n-1)\right]^{\frac{1}{2}}$, where $n$ is the number of values of $A_{0}$. Confidence intervals are tabulated here and in Tables V and VI because the standard errors do not accurately indicate the uncertainties in the $A_{0}$ values for the small $N$ values in these tables.

\begin{tabular}{|c|c|c|c|c|c|c|c|}
\hline $\begin{array}{c}P \\
\left(10^{3} \mathrm{esu}\right)\end{array}$ & $N$ & $\begin{array}{c}A_{0} \\
\left(10^{-3} \mathrm{esu}\right)\end{array}$ & $\begin{array}{c}\text { Standard } \\
\text { error } \\
\left(10^{-3} \text { esu }\right)\end{array}$ & $\begin{array}{c}95 \% \\
\text { confidence } \\
\text { interval }\end{array}$ & $\begin{array}{c}T-T_{0} \\
(\mathrm{mK})\end{array}$ & $\begin{array}{c}\text { Standard } \\
\text { error } \\
(\mathrm{mK})\end{array}$ & $\begin{array}{c}95 \% \\
\text { confidence } \\
\text { interval }\end{array}$ \\
\hline 0.621 & 7 & 3.91 & 0.05 & $0.13^{\circ}$ & 9.8 & 3.0 & 7.7 \\
\hline 1.194 & 8 & 3.90 & 0.047 & 0.12 & 4.9 & 2.8 & 6.9 \\
\hline $2.388^{\circ}$ & 6 & 4.07 & 0.06 & 0.17 & 27.2 & 2.8 & 7.8 \\
\hline 3.582 & 5 & 4.21 & 0.029 & 0.09 & 47.3 & 0.9 & 2.7 \\
\hline 4.776 & 4 & 3.95 & 0.071 & 0.31 & 41.9 & 7.7 & 22.8 \\
\hline 5.97 & 16 & 3.52 & 0.144 & 0.31 & 21.0 & 4.3 & 9.1 \\
\hline \multirow[t]{2}{*}{7.167} & 9 & 4.09 & 0.375 & 0.89 & -12.5 & 6.7 & 15.8 \\
\hline & $\overline{A_{0}}$ & $=4.01$ & $\Delta=0.13$ & 0.19 & & & \\
\hline
\end{tabular}


TABLE V. Data for sample No. 2 at $1.00 \mathrm{kbar}$.

\begin{tabular}{|c|c|c|c|c|c|c|c|}
\hline $\begin{array}{c}P \\
\left(10^{3} \mathrm{esu}\right)\end{array}$ & $N$ & $\begin{array}{c}A_{0} \\
\left(10^{-3} \mathrm{esu}\right)\end{array}$ & $\begin{array}{c}\text { Standard } \\
\text { error } \\
\left(10^{-3} \text { esu }\right)\end{array}$ & $\begin{array}{c}95 \% \\
\text { confidence } \\
\text { interval }\end{array}$ & $\begin{array}{c}T-T_{0} \\
(\mathrm{mK})\end{array}$ & $\begin{array}{l}\text { Standard } \\
\text { error } \\
(\mathrm{mK})\end{array}$ & $\begin{array}{c}95 \% \\
\text { confidence } \\
\text { interval }\end{array}$ \\
\hline \multicolumn{8}{|c|}{ Decreasing temperature } \\
\hline 1.00 & 12 & 3.62 & 0.062 & 0.14 & 2.7 & 4.2 & 9.4 \\
\hline 2.00 & 10 & 3.61 & 0.081 & 0.19 & 7.6 & 5.5 & 12.7 \\
\hline 3.00 & 6 & 3.81 & 0.15 & 0.42 & 21.4 & 6.7 & 18.6 \\
\hline 4.00 & 4 & 3.80 & 0.14 & 0.60 & 23.2 & 4.5 & 19.4 \\
\hline \multicolumn{8}{|c|}{ Increasing temperature } \\
\hline 1.00 & 8 & 3.62 & 0.084 & 0.21 & 3.1 & 3.6 & 8.8 \\
\hline 2.00 & 6 & 3.56 & 0.114 & 0.32 & 7.6 & 4.5 & 12.5 \\
\hline 3.00 & 6 & 3.74 & 0.12 & 0.33 & 20.5 & 4.5 & 12.5 \\
\hline $4: 00$ & 6 & 3.60 & 0.05 & 0.14 & 15.6 & 2.0 & 5.6 \\
\hline 5.00 & 9 & 3.52 & 0.064 & 0.15 & -2.7 & 2.2 & 5.2 \\
\hline 6.00 & 8 & 3.56 & 0.165 & 0.16 & -43.7 & 1.8 & 4.3 \\
\hline \multirow[t]{2}{*}{7.167} & \multicolumn{2}{|c|}{ Not calculated } & \multicolumn{2}{|c|}{ assuming $\vec{A}_{0}$} & -147.5 & NA & NA \\
\hline & & $=3.64$ & $\Delta=0.11$ & 0.08 & & & \\
\hline
\end{tabular}

$p-T-E$ space in which we made measurements. To determine which of our data points fall in the critical region and which in the tricritical region we examine the behavior of the critical exponents which can be derived from our data.

The exponents $\beta, \gamma$, and $\delta$ are associated with dielectric properties. However, we cannot determine $\beta$ from our macroscopic dielectric measurements because it depends on the value of $P$ in the stable phase near first-order transition surfaces in $p-T-E$ space, and near such surfaces our results correspond to an average of $P$ over the crystal, part of which is in the stable phase and part in metastable phases, as discussed in Sec. IV.

We now examine predictions of Landau theory for the critical exponents $\gamma$ and $\delta$ based on expansion of the free energy $F$ in terms of the order parameter, $P-P_{\mathrm{cr}}$. We truncate $F$ in Eq. (1) after the
$P^{6}$ term. To locate the critical lines we evaluate the derivatives

$$
\begin{aligned}
& \partial F / \partial P=E=P\left(A+B P^{2}+C P^{4}\right), \\
& \partial^{2} F / \partial P^{2}=\left(\chi_{T}\right)^{-1}=A+3 B P^{2}+5 C P^{4}, \\
& \partial^{3} F / \partial P^{3}=2 P\left(3 B+10 C P^{2}\right), \\
& \partial^{4} F / \partial P^{4}=6\left(B+10 C P^{2}\right),
\end{aligned}
$$

and note that the conditions that the susceptibility be infinite at critical points and positive everywhere require that at critical points $\partial^{2} F / \partial P^{2}=\partial^{3} F /$ $\partial P^{3}=0$ and $\partial^{4} F / \partial P^{4} \geqslant 0$. These conditions give a central critical point at $E_{\mathrm{cr}}=P_{\mathrm{cr}}=A_{\mathrm{cr}}=0$ if $B>0$, and give wing critical points at $E_{\mathrm{cr}}=8 C P_{\mathrm{cr}}^{5} / 3, P_{\mathrm{cr}}$ $= \pm(-3 B / 10 C)^{1 / 2}, A_{\text {cr }}=9 B^{2} / 20 C$ if $B<0$. Both the

\begin{tabular}{|c|c|c|c|c|c|c|c|}
\hline $\begin{array}{c}P \\
\left(10^{3} \mathrm{esu}\right)\end{array}$ & $N$ & $\begin{array}{c}A_{0} \\
\left(10^{-3} \mathrm{esu}\right)\end{array}$ & $\begin{array}{c}\text { Standard } \\
\text { error } \\
\left(10^{-3} \mathrm{esu}\right)\end{array}$ & $\begin{array}{c}95 \% \\
\text { confidence } \\
\text { interval }\end{array}$ & $\begin{array}{c}T-T_{0} \\
(\mathrm{mK})\end{array}$ & $\begin{array}{l}\text { Standard } \\
\text { error } \\
(\mathrm{mK})\end{array}$ & $\begin{array}{c}95 \% \\
\text { confidence } \\
\text { interval }\end{array}$ \\
\hline 0.50 & 14 & 4.23 & 0.085 & 0.19 & +10.6 & 5.8 & 12.6 \\
\hline 1.00 & 15 & 4.13 & 0.071 & 0.15 & -2.4 & 4.8 & 10.4 \\
\hline 2.00 & 16 & 4.09 & 0.054 & 0.12 & -15.8 & 3.8 & 8.1 \\
\hline 3.00 & 16 & 4.10 & 0.054 & 0.12 & -30.0 & 4.0 & 8.6 \\
\hline 4.00 & 9 & 3.90 & 0.085 & 0.20 & -82.4 & 5.2 & 12.3 \\
\hline 5.00 & 5 & 3.74 & 0.039 & 0.12 & -169 & 2.4 & 7.0 \\
\hline \multirow[t]{2}{*}{6.00} & 3 & 3.53 & 0.106 & 1.35 & -309 & 8.0 & 101.0 \\
\hline & $\overline{A_{0}}$ & $=4.04$ & $\Delta=0.18$ & 0.20 & & & \\
\hline
\end{tabular}
central and wing critical points found by this procedure are stable according to the bitangent con-

TABLE VI. Data for sample No. 2 at $3.00 \mathrm{kbar}$. 

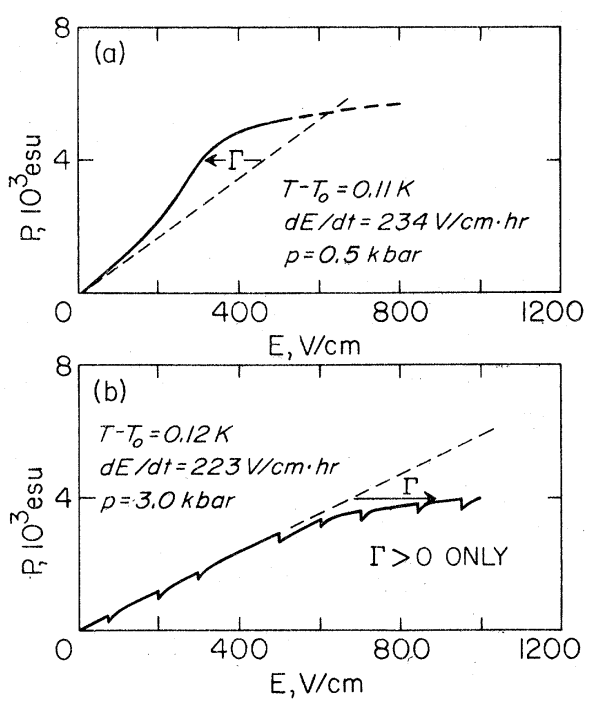

FIG. 6. Polarization vs electric field at constant temperature, at an electric field sweep rate of $\sim 230$ $\mathrm{V} \mathrm{cm}{ }^{-1} \mathrm{~h}^{-1}$. At $0.5 \mathrm{kbar}$ (a) the maximum dielectric constant occurs at $\sim 300 \mathrm{~V} \mathrm{~cm}^{-1}$, while at $3 \mathrm{kbar}(\mathrm{b})$ the dielectric constant is a maximum at $E=0$. Spikes in 3-kbar trace are due to pressure pumps. Here $\Gamma=E$ $-A_{0}\left(T-T_{0}\right) P=B P^{3}+C P^{5}$.

struction criterion. ${ }^{39}$ Because $A$ and $B$ depend on temperature $T$ and pressure $p$, three lines consisting of central and wing critical points can be constructed in $p-T-E$ space, and these lines meet at the tricritical point, which is characterized by $E_{t}=P_{t}=A_{t}=B_{t}=0$.

A Taylor expansion of the free energy about a critical point using Eqs. (9) yields

$$
\begin{aligned}
F= & F_{\mathrm{cr}}+\left[\left(A-A_{\mathrm{cr}}\right) P_{\mathrm{cr}}+E_{\mathrm{cr}}\right]\left(P-P_{\mathrm{cr}}\right) \\
& +\frac{1}{2}\left(A-A_{\mathrm{cr}}\right)\left(P-P_{\mathrm{cr}}\right)^{2} \\
& +\frac{1}{4}\left(B+10 C P_{\mathrm{cr}}^{2}\right)\left(P-P_{\mathrm{cr}}\right)^{4} \\
& +C P_{\mathrm{cr}}\left(P-P_{\mathrm{cr}}\right)^{5}+\frac{1}{6} C\left(P-P_{\mathrm{cr}}\right)^{6} .
\end{aligned}
$$

We now examine predictions for the exponents $\gamma$ and $\delta$ based on this expansion.

The exponent $\gamma$ is defined by

$$
\chi_{T}=\left(\partial^{2} F / \partial P^{2}\right)^{-1}=\left(A+3 B P^{2}+5 C P^{4}\right)^{-1} \propto \epsilon^{-\gamma},
$$

where $\epsilon=\left(T-T_{\mathrm{cr}}\right) / T_{\mathrm{cr}}$. For the central critical line, $\chi_{T}$ is evaluated for $E=P=0, A>0$. Since $A=A_{0}\left(T-T_{0}\right)$ in lowest order, and $T_{0}=T_{\mathrm{cr}}$ for this critical line, the exponent $\gamma=1$ is obtained. To arrive at an analogous definition of $\gamma$ for the wing critical lines, $\chi_{T}$ must be evaluated for $P=P_{\text {cr }}$, $A>A_{\text {cr }}$, and again $\gamma=1$ is obtained. [Note that the requirement $P=P_{\mathrm{cr}}$ used in evaluating $E=\partial F /$ $\partial\left(P-P_{\mathrm{cr}}\right)$ from Eq. (9) yields $E=E_{\mathrm{cr}}+\left(A-A_{\mathrm{cr}}\right) P_{\mathrm{cr}}$, so that $E$ and $T$ must be varied simultaneously in finding the temperature dependence of the susceptibility as needed to evaluate $\gamma$.] At the tricritical point the relation $\gamma=1$ is still obtained, so that a distinction between critical and tricritical regions cannot be made on the basis of $\gamma$.

The exponent $\delta$ is defined by $E=\partial F / \partial P \propto P^{6}$. For the central critical line, $E$ is evaluated for $T=T_{\text {cr }}$ $\left(A=A_{\mathrm{cr}}=0\right)$, so that $E=B P^{3}+C P^{5}$, yielding $\delta=3$. A corresponding definition of $\delta$ for the wing critical lines requires that $E$ be evaluated for $A=A_{\text {cr }}$, yielding

$$
\begin{aligned}
E-E_{\mathrm{cr}}= & -2 B\left(P-P_{\mathrm{cr}}\right)^{3} \pm(-15 B C / 2)^{1 / 2}\left(P-P_{\mathrm{cr}}\right)^{4} \\
& +C\left(P-P_{\mathrm{cr}}\right)^{5}
\end{aligned}
$$

as obtained by substituting $P_{\mathrm{cr}}= \pm(-3 B / 10 C)^{1 / 2}$ into $E=\partial F / \partial\left(P-P_{\mathrm{cr}}\right)$, using Eq. (10) for $F$. Again, $\delta=3$ is obtained, but the lack of symmetry near the wing critical points is reflected by the $\left(P-P_{\mathrm{cr}}\right)^{4}$. term whose sign depends on whether $E$ is above or below $E_{\text {cr }}$. At the tricritical point we have $B=0$, so $E=C P^{5}$ and $\delta=5$. If crossover from $\delta=3$ to $\delta=5$ is defined to occur where the $C\left(P-P_{\mathrm{cr}}\right)^{5}$ term is as large as the other terms(s), then crossover occurs for $\left(P-P_{\text {cr }}\right) \propto B^{1 / 2}$ or, equivalently, for

TABLE VII. Data for sample No. 3 at $0.001 \mathrm{kbar}$.

\begin{tabular}{cccccc}
\hline $\begin{array}{c}P \\
\left(10^{3} \mathrm{esu}\right)\end{array}$ & $N$ & $\begin{array}{c}A_{0} \\
\left(10^{-3} \mathrm{esu}\right)\end{array}$ & $\begin{array}{c}\text { Standard error } \\
\left(10^{-3} \mathrm{esu}\right)\end{array}$ & $\begin{array}{c}T(E=0)-T_{0} \\
(\mathrm{mK})\end{array}$ & $\begin{array}{c}\text { Standard error } \\
(\mathrm{mK})\end{array}$ \\
\hline 0.50 & 39 & 3.91 & 0.05 & 1.6 & 0.9 \\
1.00 & 41 & 3.91 & 0.05 & 3.6 & 0.8 \\
2.00 & 39 & 3.87 & 0.05 & 10.5 & 0.8 \\
3.00 & 34 & 3.92 & 0.07 & 26.4 & 1.9 \\
4.00 & 25 & 3.92 & 0.10 & 32.5 & 1.2 \\
5.00 & 22 & 4.10 & 0.16 & 34.1 & 0.9 \\
6.00 & 28 & 4.10 & 0.16 & 10.0 & 2.9 \\
7.00 & 10 & 3.70 & 0.43 & -38.0 & \\
& & & & & \\
& & $\overline{A_{0}}=3.93 \quad \Delta=0.13$ & & \\
\hline \hline
\end{tabular}


TABLE VIII. Data for sample No. 3 at 2.00 kbar. The data have been adjusted to compensate for an apparent rise in $T_{0}$ of $4.7 \mathrm{mK} /$ day during this 20 -day run.

\begin{tabular}{cccccc}
\hline $\begin{array}{c}P \\
\left(10^{3} \mathrm{esu}\right)\end{array}$ & $N$ & $\begin{array}{c}A_{0} \\
\left(10^{-3} \mathrm{esu}\right)\end{array}$ & $\begin{array}{c}\text { Standard error } \\
\left(10^{-3} \mathrm{esu}\right)\end{array}$ & $\begin{array}{c}T(E=0)-T_{0} \\
(\mathrm{mK})\end{array}$ & $\begin{array}{c}\text { Standard error } \\
(\mathrm{mK})\end{array}$ \\
\hline 0.50 & 24 & 3.97 & 0.08 & -1.5 & 3.7 \\
1.00 & 23 & 4.05 & 0.07 & 4.5 & 3.3 \\
2.00 & 23 & 3.98 & 0.08 & 2.6 & 3.7 \\
3.00 & 22 & 4.04 & 0.07 & -12.2 & 3.2 \\
4.00 & 20 & 4.02 & 0.10 & -57.6 & 3.3 \\
5.00 & 14 & 3.63 & 0.08 & -122.0 & 7.9 \\
6.00 & 9 & 3.62 & 0.20 & -290.1 & 72.1 \\
7.00 & 5 & 2.57 & 0.88 & & \\
& & & & & \\
\end{tabular}

$\left(E-E_{\mathrm{cr}}\right) \propto B^{5 / 2}$, evaluated on the $A=A_{\text {cr }}$ surface on which $\delta$ is defined. Because $B \propto\left(p-p_{t}\right)$ in lowest order, these crossover exponents occur also in the relations

$\left|P-P_{\mathrm{cr}}\right| \propto\left|p-p_{t}\right|^{1 / 2}, \quad\left|E-E_{\mathrm{cr}}\right| \propto\left|p-p_{t}\right|^{5 / 2}$, both above and below the tricritical pressure $p_{t}$.

We now present comparisons of these predictions for $\gamma$ and $\delta$ with our experimental results. We approximated $\chi_{T}=(\partial P / \partial E)_{P=0, T}$, which appears in the definition of $\gamma$, by $P / E$ for the lowest $P(500$ esu) isopol for the $3-\mathrm{kbar}$ data, because for this pressure the isopol extends all the way to $E=0$,

TABLE IX. Data for sample No. 3 at 2.40 kbar.

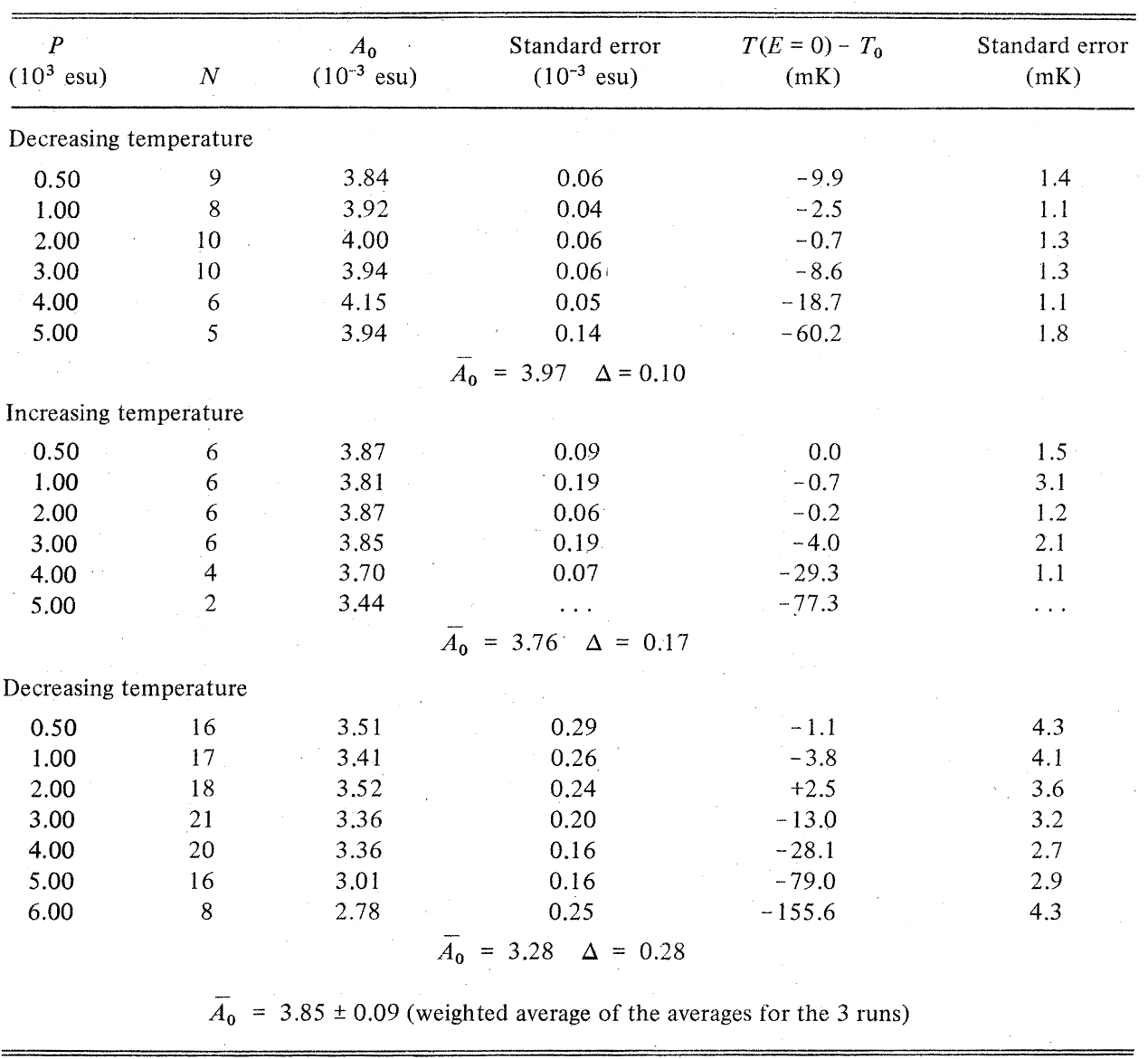


TABLE X. Landau coefficients for three $\mathrm{KH}_{2} \mathrm{PO}_{4}$ crystals at various hydrostatic pressures. The $A_{0}$ values listed below are averages weighted by the inverse squares of the standard errors for individual isopols given in Tables IV - IX, and accordingly differ somewhat from the unweighted $\bar{A}_{0}$ averages listed in those Tables.

\begin{tabular}{lcccc}
\hline $\begin{array}{c}P \\
(\mathrm{kbar})\end{array}$ & Sample No. & $\begin{array}{c}A_{0} \\
\left(10^{-3} \mathrm{esu}\right)\end{array}$ & $\begin{array}{c}B \\
\left(10^{-11} \text { esu }\right)\end{array}$ & $\begin{array}{c}C \\
\left(10^{-19} \text { esu }\right)\end{array}$ \\
\hline 0.001 & 1 & $4.3 \pm 0.2$ & $-2.35 \pm 0.40$ & $5.9 \pm 1.5$ \\
0.0016 & 2 & $3.93 \pm 0.07$ & $-1.48 \pm 0.05$ & $3.1 \pm 0.2$ \\
0.001 & 3 & $3.91 \pm 0.03$ & $-1.26 \pm 0.05$ & $3.18 \pm 0.13$ \\
1.00 & 2 & $3.64 \pm 0.08$ & $-0.89 \pm 0.05$ & $3.6 \pm 0.2$ \\
2.00 & 3 & $3.90 \pm 0.19$ & $-0.64 \pm 0.06$ & $5.88 \pm 0.14$ \\
2.40 & 3 (run 1) & $3.98 \pm 0.03$ & $-0.02 \pm 0.08$ & $4.2 \pm 0.3$ \\
2.40 & 3 (run 2) & $3.80 \pm 0.05$ & $-0.31 \pm 0.36$ & $8.9 \pm 1.7$ \\
2.40 & 3 (run 3) & $3.30 \pm 0.08$ & $+0.03 \pm 0.24$ & $4.4 \pm 0.6$ \\
2.40 & 3 (wtd. av.) & $3.89 \pm 0.03$ & $-0.03 \pm 0.07$ & $4.4 \pm 0.3$ \\
3.00 & 2 & $4.04 \pm 0.10$ & $+0.90 \pm 0.05$ & $6.1 \pm 0.2$ \\
\hline \hline
\end{tabular}

$T=T_{0}$. All of the points on this isopol, as shown in Fig. 5, were used to determine $\gamma$ in the relation $\chi_{T} \propto\left[\left(T-T_{0}\right) / T_{0}\right]^{-\gamma}$. With $T_{0}$ set at the value obtained from a best fit to all of the isopols, the value $\gamma=1.03 \pm 0.04$ resulted. With $T_{0}$ and $\gamma$ both allowed to vary to give the best fit to the 500-esu isopol, the value $\gamma=1.01 \pm 0.02$ was obtained. Accordingly, the value of $\gamma$ obtained from that data most suited for its determination shows no significant deviation from the mean-field value of 1 .

To determine $\delta$, we analyzed $E\left(P, T=T_{\mathrm{cr}}\right)$ for the data from samples 2 and 3 . Because the experimental points show no systematic deviation

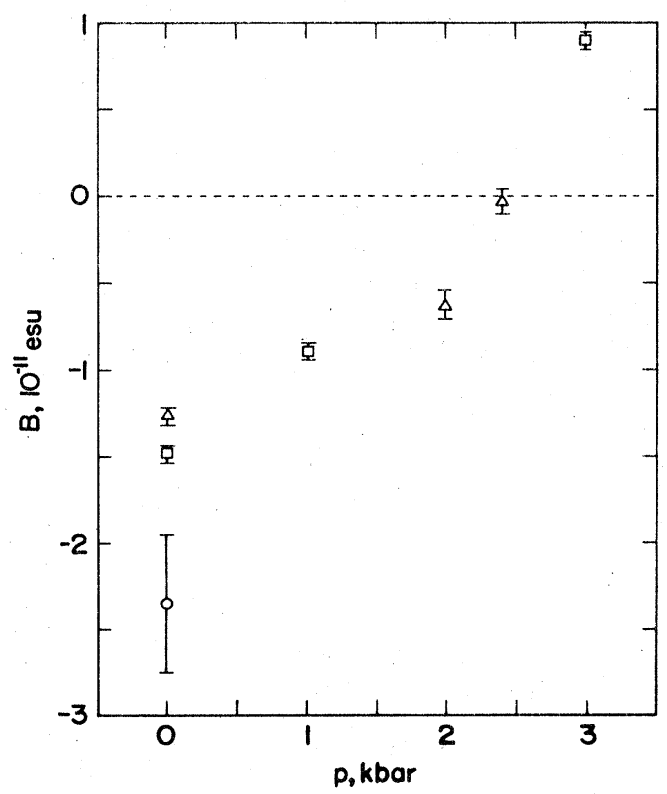

FIG. 7. Plot of Landau $B$ coefficient vs pressure $p$ for $\mathrm{KH}_{2} \mathrm{PO}_{4}$ sample 1 (circle), sample 2 (squares), and sample 3 (triangles). from straight lines, we determined $E$ from the equation of state:

$$
E=A_{0}\left(T-T_{0}\right) P+B P^{3}+C P^{5},
$$

in which $A_{0}$ and $T-T_{0}$ are best fit values for the slope and intercept of each individual isopol as given in Tables IV-IX. The uncertainties for these $E$ values were calculated from the uncertainties for $A_{0}$ and $T-T_{0}$ given in those tables.

The results of this analysis for $p=3 \mathrm{kbar}$ are shown in Fig. 8. The reliable data points all fall in the crossover region. Similar plots (not shown) for the 0 and $1 \mathrm{kbar}$ results consist of points which

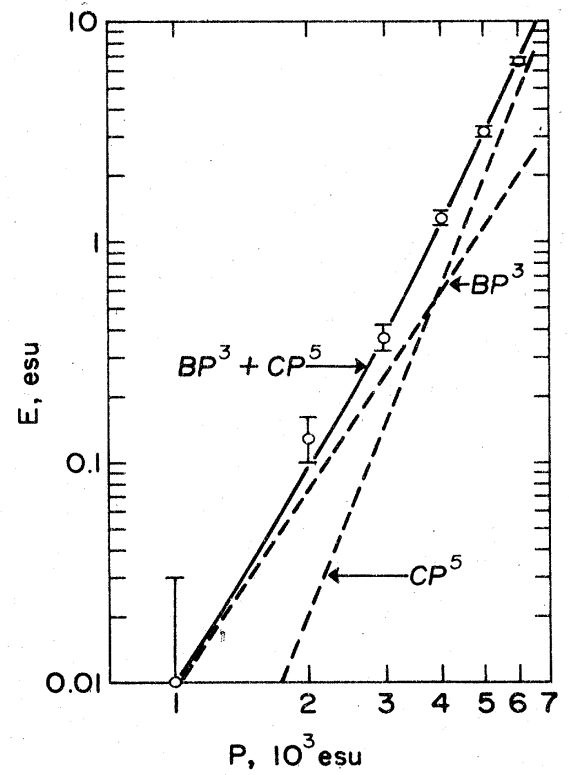

FIG. 8. Comparison of experimental points for determining critical exponent $\delta$ for $\mathrm{KH}_{2} \mathrm{PO}_{4}$ at $3 \mathrm{kbar}$, with theoretical expression (solid line) and its component terms (dashed lines) obtained from best fit to Landau theory. 


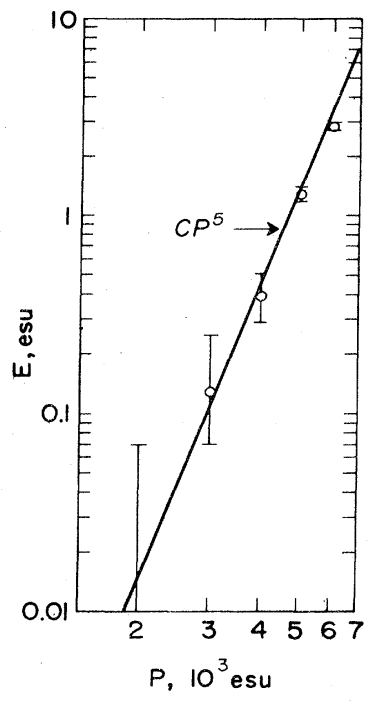

FIG. 9. Comparison of experimental points for determining critical exponent $\delta$ for $\mathrm{KH}_{2} \mathrm{PO}_{4}$ at 2.4 kbar, with theoretical expression $C P^{5}$ using $C$ from Table X. Points represent fields for which $T=T_{0}$ for isopols in third run, Table IX.

also fall in the crossover region and show similarly good agreement with the field calculated from Eq. (12). In those plots, data points for the two branches corresponding to the "+" and "-" signs in Eq. (12) are clearly separated. Accordingly, these results for the critical exponent $\delta$ show the asymmetry of the wing critical points and the crossover from critical to tricritical behavior.

At $2 \mathrm{kbar}$ we obtained ${ }^{37}$ an effective value of 5.6 for $\delta$, slightly larger than 5 as expected for the effective value for a first-order transition near the tricritical point. At $2.4 \mathrm{kbar}$ we obtained for $\delta$ the three values $5.7 \pm 0.5,5.0 \pm 0.8$, and $4.5 \pm 0.4$ which correspond respectively to the first, second, and third run results shown in Table IX. The data points for $\delta$ obtained from the third run are shown in Fig. 9. The weighted average for the three runs is 4.9 , with a standard deviation of 0.3 which seems optimistic because it does not overlap two of the three individual run values. The agreement of these values with the Landau-theory tricritical value of $\delta=5$ is an additional indication that the tricritical point is near $2.4 \mathrm{kbar}$. These are the first measurements of the exponent $\delta$ near the tricritical point for any material.

Additional evidence for a tricritical point in KDP is provided by neutron diffraction measurements made by a group at Grenoble ${ }^{40}$ of the temperature dependence of the change $\Delta c$ of the unit cell length $c$ at two pressures. Because $\Delta c$ is proportional to the square of the spontaneous polarization $P_{s}$, they were able to determine the exponent $\beta$ defined by $P_{s} \propto\left(T_{\mathrm{cr}}-T\right)^{\beta}$. They found that $\beta$ is near the Landau-theory tricritical value of $\frac{1}{4}$ at $2 \mathrm{kbar}$, and near the Landau-theory critical value of $\frac{1}{2}$ at 3.5 kbar.

\section{SUMMARY}

We have measured the net polarization charge of two $\mathrm{KH}_{2} \mathrm{PO}_{4}$ crystals as a function of applied electric field in a $0.5-\mathrm{K}$ neighborhood of the ferroelectric transition at hydrostatic pressures of 0 , $1,2,2.4$, and $3 \mathrm{kbar}$. In each case the paraelectric region is well described by the equation of state which follows from the Landau phenomenological expression for the free energy. The transition is first order at 0 and $1 \mathrm{kbar}$, with critical fields of $183 \pm 60 \mathrm{~V} / \mathrm{cm}$ at $0 \mathrm{kbar}$ and $43 \pm 13 \mathrm{~V} / \mathrm{cm}$ at 1 $\mathrm{kbar}$, and is second order at $3 \mathrm{kbar}$.

The pressure dependence of the coefficient $B$ in the Landau equation of state $E=A_{0}\left(T-T_{0}\right) P+B P^{3}$ $+C P^{5}$ indicates a tricritical point (TCP) at $2.3 \pm 0.3$ kbar. Tricritical values predicted by Landau theory were observed by us for the exponents $\gamma$ and $\delta$ and by Vettier, Bastie, and co-workers ${ }^{40}$ for the exponent $\beta$. This TCP exists in the space of temperature, pressure, and electric field, hence the "wing" structure of the TCP is experimentally accessible.

\section{ACKNOWLEDGMENTS}

We thank J. R. Brookeman and T. A. Scott for the pressure-vessel design and for instruction in high-pressure lore. Experimental advice and assistance were supplied by R. J. Pollina in early stages of this work. Helpful discussions with $\mathrm{K}$. Okada and E. K. Riedel are gratefully acknowledged. This work was supported by the NSF through Grant No. DMR 74-13220 A01. This work was based in part on a Ph.D. thesis submitted by A. B. Western and an M.S. thesis submitted by C. R. Bacon to Montana State University.
*Present address: Department of Physics and Geophysics, Montana College of Mineral Science and Technology, Butte, Montana 59701.

$\uparrow$ Present address: EG\&G, Idaho Falls, Idaho 83401.

\$Present address: Gray Tool Co., 7135 Ardmore, Houston, Texas 77002 .

${ }^{1}$ R. B. Griffiths, Phys. Rev. Lett. 24, 715 (1970).

${ }^{2} \mathrm{~A}$ staggered field can be induced by an ordinary applied field for certain orientations of some compensated antiferromagnets, as shown by R. Alben, M. Blume, L. M. Corliss, and J. M. Hastings, Phys. Rev. B 11, 295 (1975). N. Giordano and W. P. Wolf, Phys. Rev. Lett. 39 , 342 (1977) have employed such an induced staggered field to investigate the full three-dimensional phase diagram near the tricritical point in dysprosium aluminum garnet. 
${ }^{3}$ Collected Papers of L. D. Landau, edited by D. ter Haar (Gordon and Breach, New York, 1965), pp. 193-216.

${ }^{4}$ V. H. Schmidt, Bull. Am. Phys. Soc. 19, 649 (1974).

${ }^{5}$ P. S. Peercy, Phys. Rev. Lett. 35, 1581 (1975).

${ }^{6}$ The earliest report of a first-order property of which we are aware is by P. P. Craig, Phys. Lett. 20, 140 (1966). Careful measurements by Reese (Ref. 13) in 1969 established the first-order nature of the transition.

${ }^{7}$ B. A. Strukov, M. A. Korzhuev, A. Baddur, and V. A. Koptsik, Fiz. Tverd. Tela (Leningrad) 13, 1872 (1971) [Sov. Phys. Solid State 13, 1569 (1972)].

${ }^{8}$ E. V. Sidnenko and V. V. Gladkii, Kristallografiya 18,

138 (1973) [Sov. Phys. Crystallogr. 18, 83 (1973)].

${ }^{9}$ M. Vallade, Phys. Rev: B 12, 3755 (1975).

${ }^{10} \mathrm{H}$. Sugié, K. Okada, and K. Kan'no, J. Phys. Soc. Jpn. 33, 1727 (1972).

${ }_{11} \mathrm{~K}$. Okada, H. Sugié, and K. Kan'no, Phys. Lett. 44A, 59 (1973).

${ }^{12} \mathrm{~K}$. Okada and H. Sugié, Ferroelectrics 17, 325 (1977).

${ }^{13}$ W. Reese and L. F. May, Phys. Rev. 162, 510 (1967);

W. Reese, ibid. 181, 905 (1969).

${ }^{14} \mathrm{~J}$. W. Benepe and W. Reese, Phys. Rev. B $\underline{3}, 3032$ (1971).

${ }^{15}$ J. Kobayashi, Y. Uesu, and Y. Enomoto, Phys. Status Solidi B 45,293 (1971).

${ }^{16}$ T. Matsuda and R. Abe, J. Phys. Soc. Jpn. $\underline{36}, 765$ (1973).

${ }^{17} \mathrm{~J}$. Eberhard and P. Horn, Solid State Commun. 16, 1343 (1975).

${ }^{18}$ A . B. Western and V. H. Schmidt, Solid State Commun. 19,885 (1976).

${ }^{19}$ A. B. Western, A. G. Baker, R. J. Pollina, and V. H. Schmidt, Ferroelectrics 17, 333 (1977).

${ }^{20}$ V. H. Schmidt, A. B. Western, and A. G. Baker, Phys. Rev. Lett. 37, 839 (1976).

${ }^{21}$ R. T. Birge, Rev. Mod. Phys. 19, 298 (1947).

${ }^{22}$ H. Umebayashi, B. C. Frazer, G. Shirane, and W. B. Daniels, Solid State Commun. $\underline{5}, 591$ (1967).

${ }^{23} \mathrm{E}$. Hegenbarth and S. Ullwer, Cryogenics $\underline{7}, 306$ (1967).

${ }^{24}$ G. Samara, Phys. Rev. Lett. 27, 103 (1971).

${ }^{25}$ Cleveland Crystals, Inc., Box 3157 , Cleveland, Ohio,
44117.

${ }^{26}$ Lake Shore Cryotronics, Inc., 9631 Sandrock Road, Eden, N. Y. 14057.

${ }^{27}$ Flexible Silver Coating No. 16, Hanovia Liquid Gold Division, Englehard Industries, Inc., East Newark, N. J. 07102.

${ }^{28} \mathrm{P}$. Bornarel, A. Fousková, P. Guyon, and J. Lajzerowicz, Proceedings of the International Meeting on Ferroelectricity (Inst. Phys. Czech. Acad. Sci., Prague, 1966), Vol. II, p. 81.

${ }^{29}$ Berylco-25, Kawecki Berylco Industries, Inc., $220 \mathrm{E}$. 42nd St., New York, N. Y. 10017.

${ }^{30}$ Model CSC 400 Cryogenic Capacitance Controller, Lake Shore Cryotronics, Inc. (see Ref. 26).

${ }^{31}$ W. N. Lawless, Rev. Sci. Instrum. 42, 561 (1971); ibid. $\underline{46}, 625$ (1975).

${ }^{32}$ F. A. Laws, Electrical Measurements (McGraw-Hill, New York, 1917), Chap. IV, pp. 175-177.

${ }^{33}$ Before measurements on sample 3 were begun, P. S. Peercy of Sandia Laboratories kindly repaired this leak by forcing Isopar $H$ hydraulic fluid into the cone seal of the manganin cell at a pressure of $10 \mathrm{kbar}$.

${ }^{34} \mathrm{CARY} 401$ Vibrating Reed Electrometer, Cary Instruments, 2724 South Peck Road, Monrovia, Calif. 91016.

${ }^{35} \mathrm{~A}$. B. Western, Jr., Ph.D. thesis (Montana State University, Bozeman)( unpublished), available through Xerox Microfilms, Ann Arbor, Mich. 48105.

${ }^{36}$ C. R. Bacon, M.S. thesis (Montana State University, Bozeman) (unpublished).

${ }^{37}$ Preliminary values for sample 3 at $2 \mathrm{kbar}$ were reported by A. G. Baker, C. R. Bacon, and V. H. Schmidt, Bull. A m. Phys. Soc. 22, 324 (1977).

${ }^{38} \mathrm{~S}$. Torstveit (private communication).

${ }^{39} \mathrm{~A}$ description of this criterion appears in J. M. Kincaid and E. G. D. Cohen, Phys. Rep. C 22, 57 (1975). These authors also illustrate additional phenomena which can occur if the $\boldsymbol{P}^{8}$ term is retained.

${ }^{40} \mathrm{C}$. Vettier, P. Bastie, and M. Vallade, in High Pressure and Low Temperature Physics (Plenum, New York, to be published); P. Bastie, M. Vallade, C. Vettier, and C. M. E. Zeyen, Ferroelectrics (to be published); P. Bastie, M. Vallade, C. Vettier, and C. M. E. Zeyen, Phys. Rev. Lett. $\underline{40}, 337$ (1978). 